\title{
Electron donor-acceptor distance dependence of the dynamics of light-induced interfacial charge transfer in the dye-sensitization of nanocrystalline oxide semiconductors
}

\author{
Bernard Wenger $^{\dagger a, b}$, Christophe Bauer ${ }^{\ddagger a}, \mathrm{~b}$, Mohammad K. Nazeeruddin ${ }^{\mathrm{b}}$, \\ Pascal Comte ${ }^{\mathrm{b}}$, Shaik M. Zakeeruddin ${ }^{\mathrm{b}}$, Michael Grätzel ${ }^{\mathrm{b}}$, and Jacques-E. Moser*a \\ ${ }^{\mathrm{a}}$ Photochemical Dynamics Group, and ${ }^{\mathrm{b}}$ Laboratory for Photonics and Interfaces, \\ Institute of Chemical Sciences and Engineering, Ecole Polytechnique Fédérale de Lausanne, \\ CH-1015 Lausanne, Switzerland
}

\begin{abstract}
The effect of electronic and nuclear factors on the dynamics of dye-to-semiconductor electron transfer was studied employing $\mathrm{Ru}^{\mathrm{II}}$ (terpy)(NCS)$)_{3}$ sensitizers grafted onto transparent films made of titanium dioxide nanoparticles. Various approaches were strived to understand the dependence of the kinetics of charge injection and recombination processes upon the distance separating the dye molecules and the redox active surface. A series of bridged sensitizers containing $p$ phenylene spacers of various lengths and phosphonic anchoring groups were adsorbed onto $\mathrm{TiO}_{2}$ films. The kinetics of interfacial charge transfer was recorded by use of time-resolved spectroscopy in the fs-ps domain. The electron injection process was found to be biphasic with a clear exponential distance dependence of the fast kinetic component. The slower part of the kinetics was essentially unaffected by the length of the spacer bridge and was attributed to sensitizer molecules that are weakly bound to the surface with no direct contact of the anchoring group with the semiconductor. In a second approach, the kinetics of both forward- and back-electron transfer across a layer of insulating $\mathrm{Al}_{2} \mathrm{O}_{3}$ deposited onto $\mathrm{TiO}_{2}$ nanocrystalline particles was investigated. Efficient charge injection was observed over distances up to $3 \mathrm{~nm}$.
\end{abstract}

Keywords: Dye-sensitization, nanocrystalline oxide semiconductors, electron injection, light-induced interfacial electron transfer, ultrafast processes, $\mathrm{Ru}(\mathrm{II})$ polypyridyl complexes, distance dependence, core-shell nanoparticles.

\section{INTRODUCTION}

Charge injection from the excited state of a donor molecule into a continuum of electronic acceptor states in a solid has significant importance for the fundamental understanding of the dynamics of electron transfer (ET) processes. Classical theoretical treatments of ET and further quantum mechanical extensions are based on the assumption that the energies of the donor and acceptor states are matched by energy fluctuations caused by the thermal bath and thus that the overall ET kinetics is controlled by the nuclear activation barrier to achieve electronic resonance between reactant and product states. A fundamentally different situation is found in dye-sensitizer/semiconductor systems, where charge injection takes place from an excited molecular state into a wide continuum of acceptor levels constituting the conduction band of the solid. In this case, the rate constant for interfacial ET should be controlled essentially by electronic interaction, without much influence of nuclear factors. ${ }^{1,2}$

Recent ultrafast studies have shown charge injection from excited dye molecules into the conduction band of oxide semiconductors to occur in the femtosecond to picosecond time domain. ${ }^{4-19}$ The time constant for ET has been found in these studies to vary from $6 \mathrm{fs}^{7}$ to hundreds of ps. ${ }^{16-18}$ Charge transfer times $\leq 50 \mathrm{fs}$ indicate that the corresponding electronic coupling strengths are approaching the value of the thermal energy $\mathrm{k} T\left(\sim 200 \mathrm{~cm}^{-1}\right)$ and, thus, that the reactions are likely to have reached the adiabatic limit. ${ }^{20}$ The notion that the electron is transferred to the solid well before vibrational relaxation of the excited sensitizer has recently been confirmed in strong coupling cases by the observation of the dependence of ET kinetics upon the excitation photon energy, ${ }^{14,21-22}$ and that of oscillations in the transient absorption signal due to vibrational wavepacket motion during heterogeneous charge transfer. ${ }^{23,24}$

* je.moser@epfl.ch; phone 4121 693-3628; fax 4121 6934111; http://photochemistry.epfl.ch.

$\uparrow$ Present address: Optoelectronics Group, Cavendish Laboratory, University of Cambridge, Cambridge CB2 1TN, UK.

\$ Present address: Laboratory of Physical and Analytical Electrochemistry, EPFL, CH-1015 Lausanne, Switzerland.

Physical Chemistry of Interfaces and Nanomaterials V, edited by Mark Spitler, Frank Willig, Proc. of SPIE Vol. 6325, 63250V, (2006) $\cdot 0277-786$ X/06/\$15 $\cdot$ doi: 10.1117/12.681538

Proc. of SPIE Vol. 632563250 -1 
The strong electronic coupling prevailing for an efficient sensitizer is generally the result of the anchoring of the dye molecule onto the semiconductor surface through a moiety carrying its lowest unoccupied molecular orbital (LUMO) ${ }^{20}$ This situation is clearly encountered in carboxylated $\mathrm{Ru}(\mathrm{II})$ polypyridyl complexes, ${ }^{5}$ coumarin ${ }^{4}$ or alizarin ${ }^{6,7}$ dyes, for example.

A decrease of the donor-acceptor electronic coupling is likely to occur with sensitizer molecules loosely associated to the semiconductor charged surface through electrostatic interaction and/or hydrogen bonding. Results obtained for the eosinsensitized aqueous titanium dioxide colloids are exemplary of the sensitivity of the dynamics of interfacial electron transfer upon surface and environmental conditions in the weak-coupling case. ${ }^{16}$ Dispersion of dye monomers within a nanometers-thick polyvinyl-alcohol adlayer yielded a broad distribution of distances separating the sensitizer's excited states from the reactive surface. In this situation, kinetic parameters for charge injection in the conduction band of $\mathrm{TiO}_{2}$ were found to cover a large time span from typically $200 \mathrm{fs}$ to hundreds of picoseconds, and were only limited at longer times by radiative and nonradiative decay of the dye excited states.

Because of their successful use in dye-sensitized solar cells, Ru(II) polypyridyl complex dyes adsorbed on nanocrystalline $\mathrm{TiO}_{2}$ films have been regarded as model systems for the experimental study of the ultrafast dynamics of interfacial light-induced electron transfer. Most studies have reported charge injection kinetics from $\mathrm{Ru}\left(\mathrm{dcbpyH}_{2}\right)_{2}(\mathrm{NCS})_{2}$ (N3) or its partially deprotonated form (N719) to take place with a fast (sub-100 fs) phase, followed by a slower (0.7-200 ps) multi-exponential component. This complex, multiphasic behavior observed for the electron injection process has prevented the development of a satisfying kinetic model and has lead to often contradicting conclusions. Recently, we showed that the observed kinetic heterogeneity can actually result from the aggregation of sensitizer molecules on the surface. A monophasic ET with a rise time shorter than $20 \mathrm{fs}$ is indeed consistently observed when the formation of aggregates is prevented and the sensitizer is adsorbed as a monolayer on the surface of $\mathrm{TiO}_{2}$ nanocrystals. ${ }^{19}$

The examples provided by eosin- and N3-sensitization of titanium dioxide demonstrate that the mode and geometry of adsorption of dye-sensitizers at the surface of the semiconductor can strongly affect the ultrafast photoinduced charge injection dynamics. The kinetic heterogeneity of the reaction can also be rationalized by other causes: Sundström and collaborators have proposed the biphasic electron injection from $\mathrm{N} 3$ into $\mathrm{TiO}_{2}$ is due to a two-state mechanism, the fast and slow components being attributed to the injection from the singlet and triplet excited states of the ruthenium complex, respectively. ${ }^{14,15}$ Interligand electron dynamics within the dye sensitizer was also invoked as a source of additional kinetic complexity. ${ }^{25}$ Haque et al. showed that the nonexponential nature of the injection dynamics can be simulated by a simple inhomogeneous disorder model. ${ }^{18}$

The electronic coupling for ET can be diminished deliberately by increasing the distance separating the LUMO of the dye from the surface of the semiconductor material. The dependence of the multiphasic injection dynamics upon this parameter could serve to discriminate between the various possible sources of kinetic heterogeneity. This can be achieved, for instance, by inserting insulating spacer units between the chromophore and the anchoring group of the dye.

Lian and co-workers have studied the bridge-length dependence of ultrafast charge injection from rhenium-polypyridyl complexes to $\mathrm{TiO}_{2}$ and $\mathrm{SnO}_{2}$ films and have suggested that the transition between the strong- (adiabatic) and weak coupling (non-adiabatic) cases takes place for transfer distances increased by only one $-\mathrm{CH}_{2}$ unit length $(\sim 3 \AA)$. ${ }^{13,26}$ The lengthening of the bridge spacer does not lead in all instances to slower kinetics, especially if the linker is too flexible or when the molecule can adopt a tilted orientation on the surface. In an attempt to circumvent this problem, Galoppini et al. synthesized a family of rigid-rod and tripodal linkers to anchor sensitizers to the surface of semiconductor oxide. ${ }^{26}$ Sub-picosecond injection rate was observed over a distance of $24 \AA$, indicating important delocalization of the excited state over the rigid spacer arm. ${ }^{28}$ More recently, a perylene-based tripodal sensitizer was studied in conjunction with other perylene sensitizers in ultra-high vacuum conditions. ${ }^{29,30}$ Injection time constants ranging from $13 \mathrm{fs}$ to 4 ps were measured and an exponential dependence upon ET distance was observed with a damping factor $\beta=1 \AA^{-1}$.

Insulating layers of large bandgap metal oxides have been used as barriers for back electron transfer in dye-sensitized nanocrystalline solar cells. ${ }^{31,33}$ Though, no systematic study of the distance effect has been carried out on this type of system. Here, we report on the distance dependence of interfacial electron transfer by two different approaches. First, bridged sensitizers, featuring Ru-terpyridyl separated from the surface by 0,1 or 2 phenylene units, have been studied using femtosecond transient absorbance technique. Mesoscopic films constituted of $\mathrm{Al}_{2} \mathrm{O}_{3} / \mathrm{TiO}_{2}$ core-shell nanoparticles with insulating layers of controlled thickness were then used to investigate the retardation of both charge injection and back electron transfer processes. 


\section{EXPERIMENTAL}

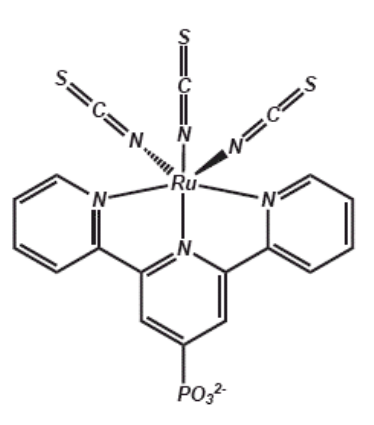

Po

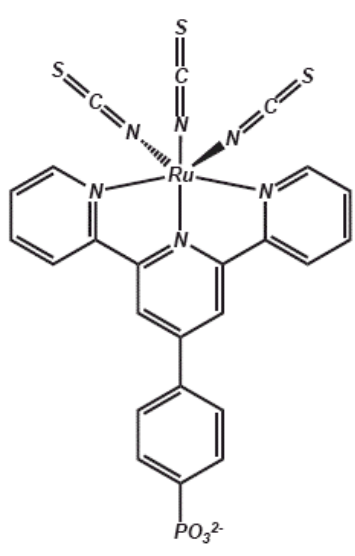

P1

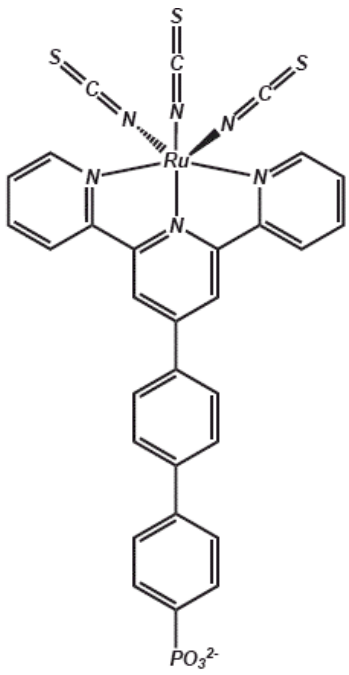

P2

Fig. 1. Phenylene-bridged phosphonated Ru(II) (terpy)(NSCS) $)_{3}$ dye sensitizers.

\subsection{Sample preparation}

Dye-sensitizers P0, P1 and P2 (Fig. 1) were synthesized according to a procedure adapted from that used for parent $\mathrm{Ru}$ (terpy)(NCS) $)_{3}$ complexes. ${ }^{34} 8 \mu \mathrm{m}$-thick pure $\mathrm{TiO}_{2}$ nanocrystalline films were prepared according to a previously published procedure. ${ }^{3}$ Core-shell particles were produced by depositing successive layers of alumina on $20 \mathrm{~nm}$-diameter anatase nanoparticles by a sol-gel technique. Nanocrystalline films were then prepared by sintering such particles together to form a mesoporous network by a method similar to that used for pure $\mathrm{TiO}_{2}$. Nanocrystalline films constituted of pure $\mathrm{TiO}_{2}$ or $\mathrm{Al}_{2} \mathrm{O}_{3} / \mathrm{TiO}_{2}$ core-shell particles were soaked overnight in the dye solutions $(\sim 0.3 \mathrm{mM}$ in EtOH$)$. After dyeing, they were rinsed with ethanol, dried and covered by a film of methoxy-propionitrile (MPN) solvent.

\subsection{Time-resolved spectroscopy}

The femtosecond pump-probe spectrometer ${ }^{16}$ employed to measure the kinetics of charge injection to the semiconductor and the nanosecond transient absorption setup ${ }^{35}$ used to monitor back electron transfer dynamics were described earlier. Ultrafast kinetics were measured in a two colors pump-probe configuration. Pump (535 nm) and probe $(570 \mathrm{~nm})$ were tuned by two NOPAs, yielding a typical cross correlation time of $50 \mathrm{fs}$. Intensities of both beams were reduced to less than $1 \mu \mathrm{J} /$ pulse and the samples were constantly translated to avoid thermal damaging of the dye-sensitized films.

\section{EXPERIMENTS AND RESULTS}

\subsection{Transient absorption spectra}

The transient absorption spectra of $\mathbf{P 0}$ in solution in ethanol and adsorbed on the surface of $\mathrm{TiO}_{2}$ have been recorded in the ns- $\mu$ s time domain. Results are displayed in Fig. 2. In solution, the transient spectrum is dominated by the strong absorption of the dye excited states, whose large extinction coefficient and broad spectrum prevent any bleaching to be observed over the entire visible domain. The lifetime of the excited state in aerated methoxy-propionitrile (MPN) solution was estimated to be $\tau=34 \mathrm{~ns}$. When $\mathbf{P 0}$ is adsorbed onto titanium dioxide nanoparticles, its photoexcited states are efficiently quenched and the bleaching of the ground state of the molecule is observed at $\lambda=550 \mathrm{~nm}$. At longer wavelengths, the spectrum exhibits a broad absorption band centered at $700 \mathrm{~nm}$, which is assigned to the oxidized state of the molecule. As expected, the phenylene units of the bridge spacers in P1 and $\mathbf{P 2}$ dyes have practically no influence on the electronic transitions involved in the absorption of light by the ground-, excited- and oxidized states and quite similar spectral data were recorded for all three compounds. 


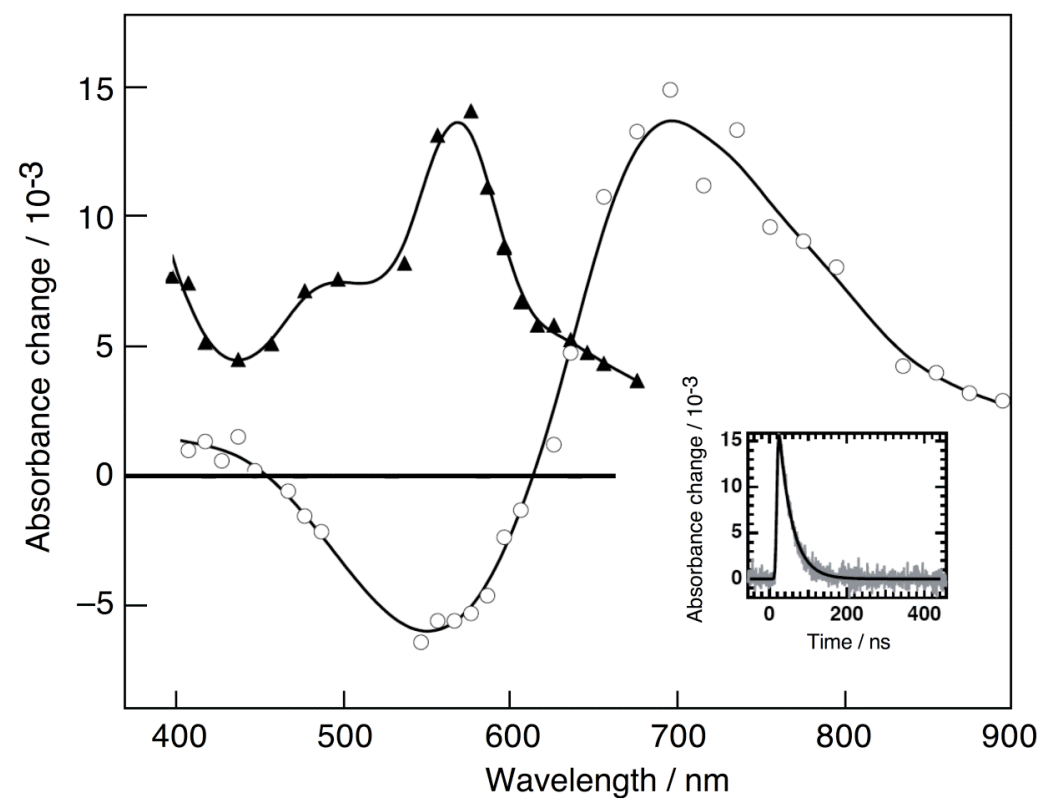

Fig. 2. Transient absorption spectra of $\mathbf{P 0}$ (Z235) dye in solution in ethanol ( $\mathbf{\Delta}$, recorded $30 \mathrm{~ns}$ after the excitation laser pulse), and adsorbed on nanocrystalline $\mathrm{TiO}_{2}(\bigcirc, 1 \mu \mathrm{s}$ after the excitation laser pulse). The inset shows the transient absorption decay of the dye excited state observed at $\lambda=570 \mathrm{~nm}$ in solution in aerated MPN ( $\tau=34 \mathrm{~ns})$.

\subsection{Injection kinetics of bridged ligand sensitizers}

Interfacial ET dynamics was probed at $\lambda=570 \mathrm{~nm}$ by monitoring the decay of the excited state absorption. Transient traces recorded for $\mathbf{P 0}, \mathbf{P 1}$ and $\mathbf{P 2}$ adsorbed on $\mathrm{TiO}_{2}$ nanocrystalline films are shown in Fig. 3. For all three sensitizers the appearance of a positive absorption signal corresponding to the build-up of the excited state was observed, followed by a fast, multiexponential decay that is attributed to the charge injection process and the formation of the dye oxidized state.

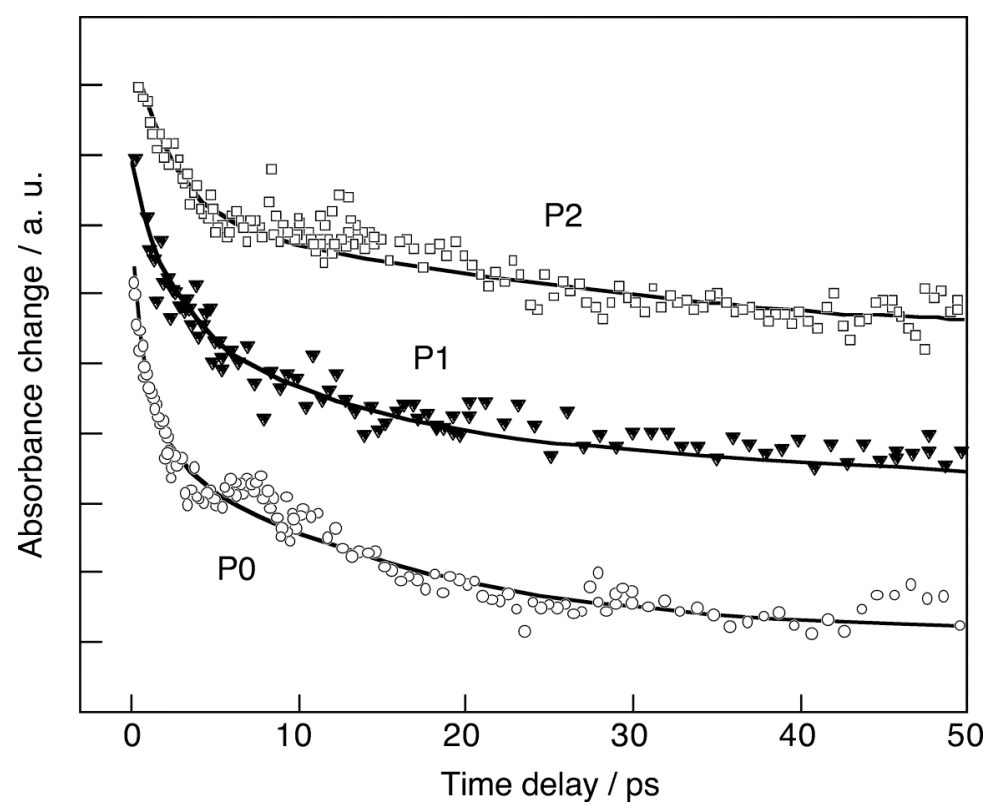

Fig. 3. Transient absorption decay of excited states of dyes P0, P1, and $\mathbf{P 2}$ adsorbed on nanocrystalline $\mathrm{TiO}_{2}$ films. Pump wavelength: $535 \mathrm{~nm}$, probe wavelength: $570 \mathrm{~nm}$. 
Data were satisfactorily fitted with double exponential decay functions, convoluted with a Gaussian instrument response. The time constants and the relative amplitudes of both exponential decays are provided in Table 1.

Table 1. Bi-exponential fitting parameters for transient absorption data reported in Fig. 3.

\begin{tabular}{|c|c|c|c|}
\hline Dye sensitizer & Ligand length $[\AA]$ ] & $\boldsymbol{\tau}_{\boldsymbol{i n j} \text { }}[\mathbf{p s}]$ (amplitude) & $\boldsymbol{\tau}_{\boldsymbol{i n j}}$ [ps] (amplitude) \\
\hline P0 & 9 & $0.9(48 \%)$ & $16(52 \%)$ \\
P1 & 13 & $1.8(39 \%)$ & $73(61 \%)$ \\
P2 & 19 & $5.8(75 \%)$ & $51(25 \%)$ \\
\hline
\end{tabular}

Assuming reorganization energy is small for all three sensitizers and does not vary much with the length of the bridge ligand, the distance dependence of the kinetics is mainly controlled by the change in the electronic coupling between the donor and the acceptor. The rate constant $k_{e t}$ should thus decrease exponentially with the transfer distance $r$ :

$$
k_{e t}=k_{0} \exp \left[-\beta\left(r-r_{0}\right)\right]
$$

The ET distance for charge injection could be represented in this case by the spatial separation of the $\pi^{*}$ orbital of the terpyridyl ligand to the nearest $\mathrm{Ti}^{4+}$ of the oxide surface. Because of delocalization of electrons in the excited state and a possible tilt angle of the spacer bridge relative to the surface normal, this distance is not trivial to estimate. Therefore, a ligand length, defined as the distance separating the $\mathrm{Ru}^{2+}$ ion from the $\mathrm{Ti}^{4+}$ ion of the oxide's surface that is directly coordinated by the phosphonic group is used here. The damping factor $\beta$ can be estimated from the slope of the semilogarithmic plot of the rate constant with respect to the ligand length (Fig. 4).

Fig. 4 shows that no clear trend is observed for the slower kinetic component, which, in light of previous observations, is attributed to ET from loosely bound or aggregated molecules. The rate constant of the fast decay component, though, follows a nicely linear trend, whose slope yields a value of $\beta=0.19 \AA^{-1}$.

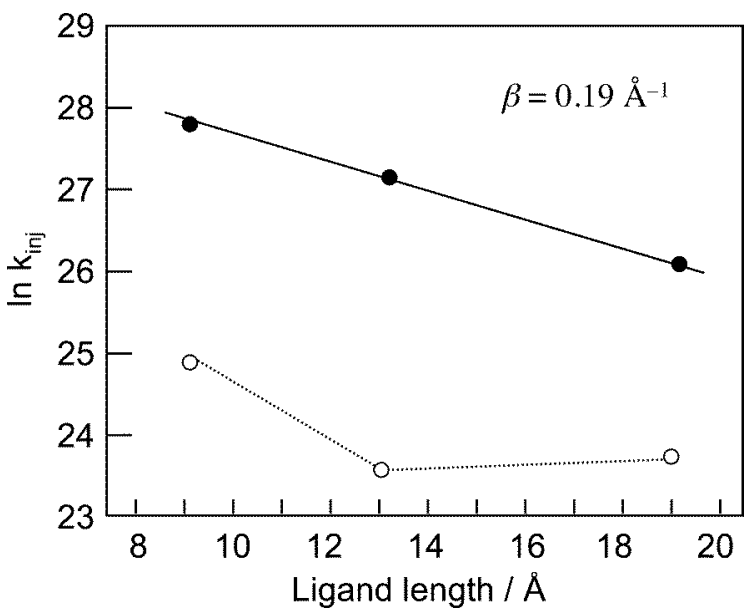

Fig. 4. Dependence of the charge injection time constant measured with P0-, P1- and P2-sensitized $\mathrm{TiO}_{2}$ nanocrystalline

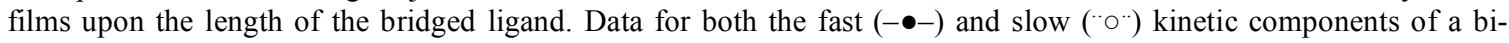
exponential fit are shown separately.

Such a small value of the damping factor $\beta$ would indicate that delocalization of electrons along the spacer bridge is effective. ${ }^{28}$ However, despite the adsorption of the dye on the surface with high density coverage, it cannot be excluded that sensitizer molecules can adopt a tilted configuration. The $\beta$ value in this case would be underestimated by a factor $\cos \theta$, where $\theta$ is the average tilt angle of the bridge axis with respect to the surface normal. 


\subsection{Injection kinetics at the surface of core-shell particles}

All nanocrystalline films constituted of core-shell particles were dyed with $\mathbf{P 0}$ sensitizer. Although the dye is now adsorbed on alumina, practically no change of its spectral characteristics compared to the adsorption onto $\mathrm{TiO}_{2}$ is observed. Transient absorption traces obtained on core-shell substrates with various alumina layer thicknesses are depicted in Fig. 6. The trace observed for the $60 \AA$ layer clearly indicates that vibrational relaxation of the dye excited state, which is expected to take place within a few picoseconds, does not lead to any significant spectral change at the probe wavelength of $560 \mathrm{~nm}$. Therefore, it can be reasonably inferred that the observed dynamics of the excited state transient absorption is due to the sole redox quenching of the photoexcited complex through charge injection in the solid. With the exception of the $60 \AA$ thick layer, all traces show an ultrafast decay with a sub-ps time constant. This component is then followed by a slower kinetic phase that is fitted with two additional exponential functions, convoluted with the same Gaussian instrument response.
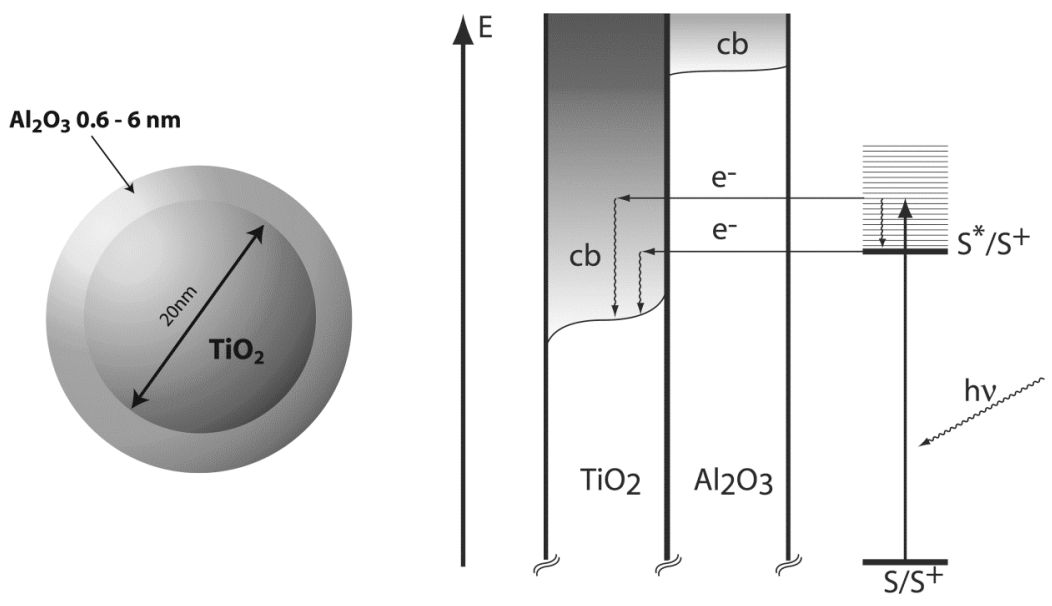

Fig. 5. $\mathrm{Al}_{2} \mathrm{O}_{3} / \mathrm{TiO}_{2}$ core-shell nanoparticles. Energetic diagram for electron injection from a dye-sensitizer excited state ( $\left.\mathrm{S}^{*}\right)$ into the conduction band of $\mathrm{TiO}_{2}$. Tunneling through the alumina barrier is required for interfacial ET to take place.

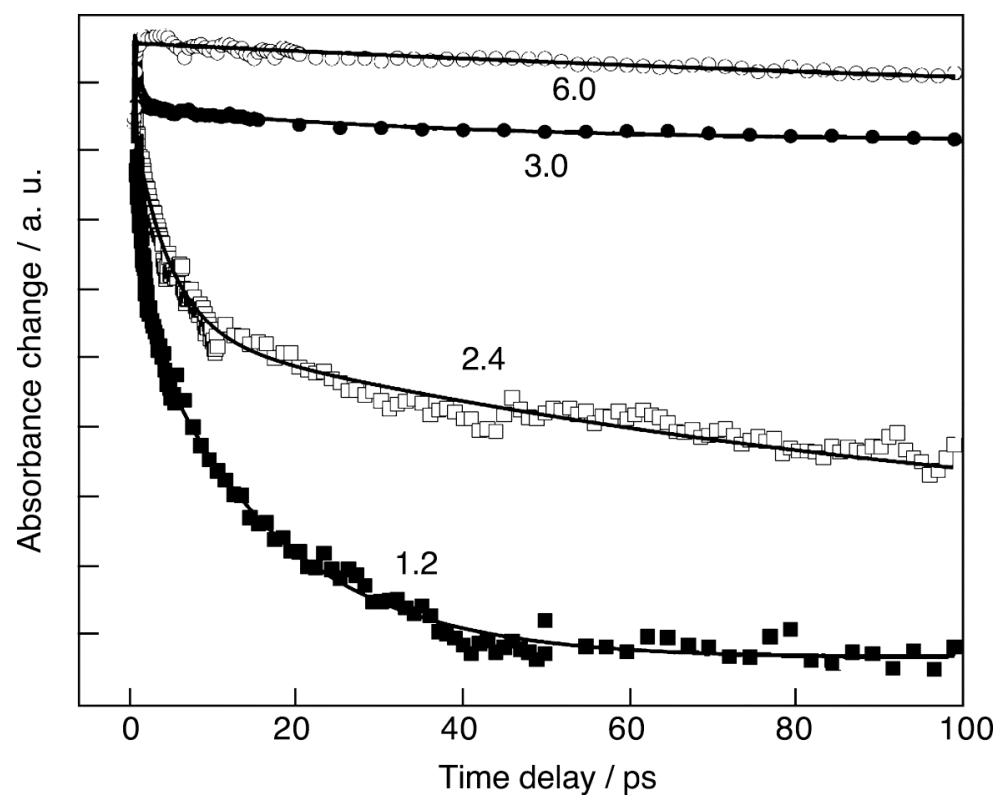

Fig. 6. Transient absorption decay of the photoexcited state of $\mathbf{P 0}$ ( $\mathrm{Z235}$ ) dye adsorbed onto $\mathrm{Al}_{2} \mathrm{O}_{3} / \mathrm{TiO}_{2}$ core-shell nanocrystalline films. Alumina layer thickness was $1.2 \mathrm{~nm}, 2.4 \mathrm{~nm}, 3.0 \mathrm{~nm}$, and $6.0 \mathrm{~nm}$. Pump wavelength: $530 \mathrm{~nm}$, probe wavelength: $560 \mathrm{~nm}$. 
Table 2. Biexponential fitting parameters for transient absorption data reported in Fig. 6.

\begin{tabular}{|c|c|c|}
\hline $\mathbf{A l}_{\mathbf{2}} \mathbf{O}_{\mathbf{3}}$ layer thickness [nm] & $\boldsymbol{\tau}_{\boldsymbol{i n j}}$ [ps] (amplitude) & $\boldsymbol{\tau}_{\text {inj }}$ [ps] (amplitude) \\
\hline 0 & $0.9(48 \%)$ & $16(52 \%)$ \\
1.2 & $1.1(28 \%)$ & $16(64 \%)$ \\
2.4 & $4.7(53 \%)$ & $71(45 \%)$ \\
3.0 & $13(67 \%)$ & $41(31 \%)$ \\
6.0 & $240(93 \%)$ & - \\
\hline
\end{tabular}

Neglecting the early sub-ps kinetic component, which is probably due to pinholes in the alumina coating and injection of dye molecules directly anchored onto the $\mathrm{TiO}_{2}$ core, a semi-logarithmic plot of the rate constants obtained from the biexponential fitting of the decay curves can again be drawn (Fig. 7). While the slow kinetic component appears to be only very weakly dependent upon the increase of the thickness of the insulating layer, a reasonably linear trend is observed for the faster kinetic phase with an estimated value of the damping factor of $\beta=0.11 \AA^{-1}$. Experimental results clearly show that electron injection occurs with a relatively high quantum yield for barriers as thick as 2-3 $\mathrm{nm}$.

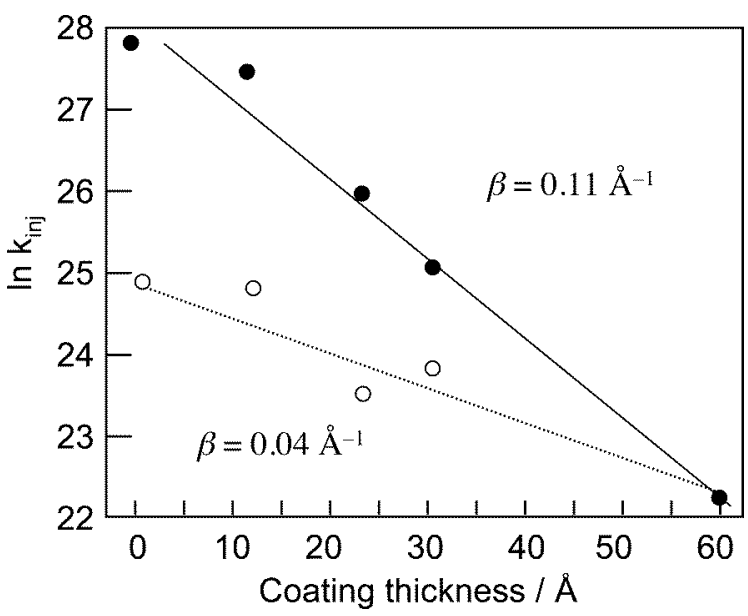

Fig. 7. Dependence of the charge injection time constant measured with P0-sensitized core-shell nanoparticles upon the

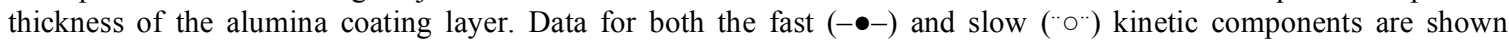
separately.

\subsection{Back electron transfer at the surface of core-shell particles}

The kinetics of the recombination reaction between the injected conduction band electrons in titanium dioxide and the oxidized dye species at the surface of the nanoparticles was studied by use of ns transient absorption spectroscopy. The decay of the absorption signal of the oxidized P0 dye observed at $\lambda=650 \mathrm{~nm}$ was monitored on nanocrystalline films containing $\mathrm{TiO}_{2}$ particles coated by alumina layers $0,6,12,15,24,30$, and $60 \AA$ thick. The excitation laser pulse $(\lambda=530 \mathrm{~nm})$ fluence was intentionally kept at a very low value $\left(\leq 25 \mu \mathrm{J} / \mathrm{cm}^{2}\right)$ to ensure that, on the average, less than one electron is injected per nanocrystalline particle upon pulsed irradiation. Under such conditions, where transport of electrons from one particle to the next one is to slow to intervene, first-order kinetics have been observed for charge recombination at Ru-complexes/ $\mathrm{TiO}_{2}$ interfaces. ${ }^{35}$ Here, the kinetics are however multiexponential and rate constants were approximated by the reciprocals of the half reaction times $\left(1 / t_{1 / 2}\right)$. Selected temporal traces displayed in Fig. 8 show that the recombination time constant varies over several orders of magnitude, spanning from $t_{1 / 2}=6 \mu$ s for bare $\mathrm{TiO}_{2}$ particles to $t_{1 / 2}=60 \mathrm{~ms}$ for $60 \AA$-thick layers of alumina.

Fig. 9 depicts the dependence of the time constants of the back electron transfer process with respect to the thickness of the alumina barrier. Again, an exponential relation was found and a damping factor of $\beta=0.15 \pm 0.01 \AA^{-1}$ could be estimated from the slope of the semi-logarithmic plot. 


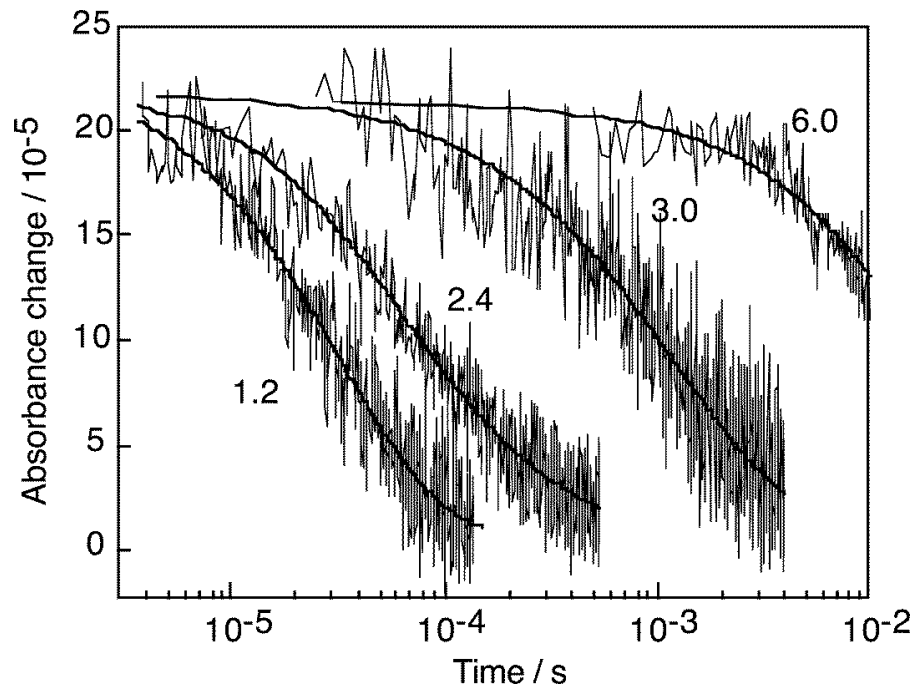

Fig. 8. Transient absorption decay of the oxidized state of $\mathbf{P 0}$ ( $\mathrm{Z235}$ ) dye adsorbed onto $\mathrm{Al}_{2} \mathrm{O}_{3} / \mathrm{TiO}_{2}$ core-shell nanocrystalline films. Alumina layer thickness was $1.2 \mathrm{~nm}, 2.4 \mathrm{~nm}, 3.0 \mathrm{~nm}$, and $6.0 \mathrm{~nm}$. Pump wavelength: $535 \mathrm{~nm}$, probe wavelength: $650 \mathrm{~nm}$. Excitation laser pulse fluence $\leq 25 \mu \mathrm{J} / \mathrm{cm}^{2}$. Traces shown are typically averaged over 3,000 laser shots.

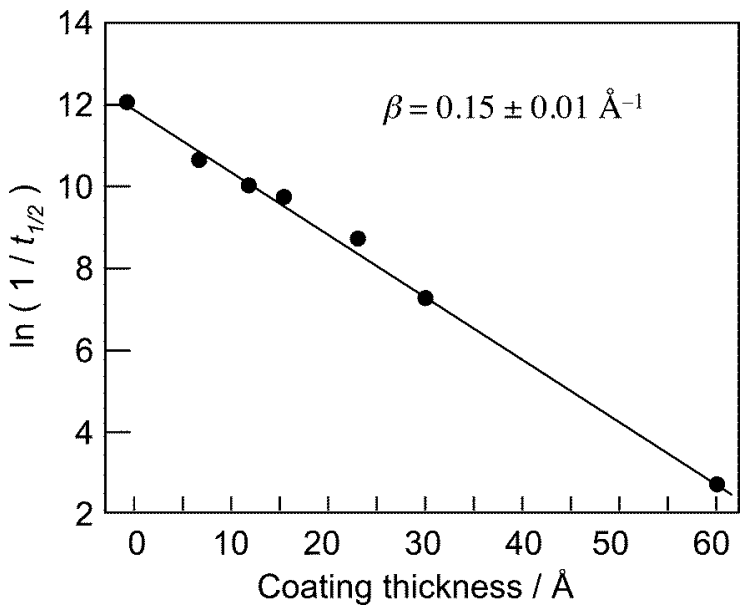

Fig. 9. Dependence of the back electron transfer time constant observed for P0-sensitized core-shell nanoparticles upon the thickness of the alumina coating layer.

\section{DISCUSSION}

The distance dependence of the electron transfer kinetics at dye/semiconductor interfaces has been studied by two different approaches: a) the use of bridged sensitizers, and b) the application of an alumina shell on $\mathrm{TiO}_{2}$ core nanoparticles. Transient absorption spectroscopy in the fs-ps and $\mu \mathrm{s}-\mathrm{ms}$ time domains made it possible to quantify the electron transfer rates for both forward and back electron transfers. The results are in good agreement with an exponential dependence of the rate constant upon distance as predicted for electron tunneling processes. The distance damping parameter $\beta$ estimated from the different experiments is close to being constant, with a value of 0.1-0.2 $\AA^{-1}$, indicative of a weak distance dependence of interfacial ET kinetic rates.

Typical damping factors for donor-bridge-acceptor systems in solution of $\beta=0.4-0.5 \AA^{-1}$ were reported for oligophenylene bridges. ${ }^{36,37}$ If an actual $\beta$ value of $0.4 \AA^{-1}$ is assumed, the decrease of the rate constant $k_{i n j}$ with the ligand 
length would imply an average tilt angle of the dye molecule of $\gamma=\cos ^{-1}(0.19 / 0.4)=62^{\circ}$ with respect to the surface normal. Such a geometry, where the sensitizers would almost have to lean flat on the oxide, is unlikely to occur in a dense packing situation, where molecules are expected to stand more or less perpendicular to the surface. Recently, DFT computational simulations of the length dependence of the electrical transmission of oligo-( $p$-phenylene) dithiolate molecular wires yielded damping factors of $0.17 \AA^{-1}$ and $0.26 \AA^{-1}$ for planar and non-planar configurations, respectively. ${ }^{38}$ These values obtained from purely electronic factors exactly predict the experimental value obtained for electron injection dynamics in our case and implicitly confirm that nuclear reorganization, contrary to donor-acceptor systems in solution, plays here a negligible role.

Retardation of both the charge injection and the back electron transfer over several order of magnitudes is achieved by inserting a wide bandgap material layer between $\mathrm{TiO}_{2}$ and the sensitizer while efficient electron injection is still observed. This result is consistent with the increase of the overall efficiency of dye-sensitized solar cells featuring coreshell nanocrystalline films. ${ }^{31-33}$ More surprising is the weak dependence of the kinetics upon the thickness of the layer. From the excellent agreement with an exponential dependence of the back electron transfer rate constant upon distance, we believe that the measured $\beta$ values are reliably describing the effect of the alumina layer on the recombination kinetics. The magnitude of the estimated damping factor $\left(\beta<0.2 \AA^{-1}\right)$ is of the same order of magnitude as that of $\pi$ conjugated systems or $\mathrm{C}_{60}$ films. ${ }^{36,37}$ In other words the conductance of what was believed to be "insulating" layers appears to be similar to that of molecular wires or electron transport layers (ETL).

The probability $P$ of electron tunneling between conductors separated by a potential barrier can be estimated from basic quantum mechanics: ${ }^{39}$

$$
P=P_{0} \exp [-2 \kappa \Delta x], \text { where } \kappa=2 m_{e} E_{B} / \hbar^{2}
$$

$P_{0}$ is here a pre-factor close to unity, $E_{B}$ is the height of the barrier potential, $m_{e}$ the electron mass, $\Delta x$ is the thickness of the barrier and $\kappa$ is a decay reciprocal length that has a meaning similar to that of the damping factor $\beta$. Bulk, stoichiometric $\mathrm{Al}_{2} \mathrm{O}_{3}$ (sapphire) has a band gap of $8.8 \mathrm{eV}$. This implies that the position of the conduction band edge is at least $3 \mathrm{eV}$ above the dye excited state energy level. ${ }^{33}$ From Eq. 2, we estimate for $E_{B}=3 \mathrm{eV}$ a value of $\beta \approx 2 \kappa=1.8 \AA^{-1}$. This very high value would imply that practically no ET is observed for a layer thickness $>7 \AA$.

Recently, it was reported, though, that in very thin tunnel layers made of amorphous aluminium oxide, the barrier height was only $1.2 \mathrm{eV}$ with respect to aluminium metal. ${ }^{40}$ This hints that the electronic structure of alumina in core-shell nanoparticles cannot be directly assumed from bulk properties. From the electron work function of $\mathrm{Al}(4.2 \mathrm{eV})$, it is inferred from the latter reference that empty states should exist in nm-sized alumina layers at a potential of $-1.5 \mathrm{~V} /$ SHE. This potential precisely corresponds to the oxidation potential of the excited state of $\mathbf{P 0}$ and therefore indicates that amorphous alumina could directly mediate electron transfer from the dye excited states to the conduction band of $\mathrm{TiO}_{2}$. Since the conduction band edge of titanium dioxide lies approximately $0.3-0.5 \mathrm{eV}$ lower than the dye excited state, ${ }^{33,34}$ back electron transfer reaction should experience a barrier height of the same order of magnitude, which would explain why the distance damping factor measured in this case $\left(0.15 \AA^{-1}\right)$ is higher than that of the injection process $\left(0.11 \AA^{-1}\right)$.

These results show that in both the bridged ligand sensitizers systems and the core-shell nanocrystalline films, the spacers used are far from being perfect insulators. Multilayer adsorption or formation of molecular aggregates on the surface of the semiconductor are likely to involve a marked increase of the interfacial ET distance. Owing to the size of the P0 dye molecule ( $>13 \AA$ ), the presence of a second layer could lead to dramatic changes in the kinetics, since the transfer distance can be lengthened in this case by more than $12 \AA$. Disordered dye molecules constituting the medium electron would have to tunnel across are expected to oppose a distance damping factor larger than that of oriented linear oligo-phenylene wires. Typically, a spatial increment of $12 \AA$ would increase the time constant by more than one order of magnitude if $\beta=0.2 \AA^{-1}$ and by a factor of 400 if $\beta=0.5 \AA^{-1}$. Inversely, the retardation of the charge injection rate by a factor 1,000 (from a time constant of $20 \mathrm{fs}$ to $20 \mathrm{ps}$, for instance) ${ }^{19}$ would imply that the distance of the excited state LUMO to the surface is typically increased by $14 \AA$ and $35 \AA$, for $\beta=0.5 \AA^{-1}$ and $0.2 \AA^{-1}$, respectively, which corresponds roughly to the thickness of 1-3 molecular layers.

Although the mechanism leading to the weak influence of distance for interfacial ET are not the same for core-shell particles as for bridged sensitizers, our experiments demonstrate that efficient charge transfer over significant distances can be achieved at dye/ semiconductor interfaces. These results encourage the use of various strategies to control electron transfer kinetics for the design of more efficient devices in the emerging field of molecular photonics. 


\section{REFERENCES}

1. J. M. Lanzafame, S. Palese, D. Wang, and R. J. D. Miller, A. A. Muenter, J. Phys. Chem. 98, 11020-11033 (1994).

2. R. J. D. Miller, G. L. McLendon, A. Nozik, W. Schmickler, and F. Willig, Surface Electron-Transfer Processes, VCH, New York, 1995.

3. M. Grätzel and J. E. Moser, Solar Energy Conversion, In Electron Transfer in Chemistry, V. Balzani, Ed., WileyVCH, Weinheim, 2001; Vol. 5, pp 589-644.

4. J. M. Rehm, G. L. McLendon, Y. Nagasawa, K. Yoshihara, J. E. Moser, and M. Grätzel, J. Phys. Chem. 100, $9577-$ 9578 (1996).

5. Y. Tachibana, J. E. Moser, M. Grätzel, D. R. Klug, and J. R. Durrant, J. Phys. Chem. 100, 20056-20062 (1996).

6. R. Huber, S. Spörlein, J. E. Moser, M. Grätzel, and J. Wachtveitl, J. Phys. Chem. B 104, 8995-9003 (2000).

7. R. Huber, J. E. Moser, M. Grätzel, and J. Wachtveitl, J. Phys. Chem. B 106, 6494-6499 (2002).

8. Y. Tachibana, S. A. Haque, I. P. Mercer, J. E. Moser, D. R. Klug, and J. R. Durrant, J. Phys. Chem. B, 105, 7424$7431(2001)$.

9. B. Burfeindt, T. Hannappel, W. Storck, and F. Willig, J. Phys. Chem. 100, 16463-16465 (1996).

10. M. Hilgendorff, and V. Sundström, J. Phys. Chem. B 102, 10505-10514 (1998).

11. I. Martini, J. H. Hodak, and G. V. Hartland, J. Phys. Chem. B 102, 9508-9517 (1998).

12. J. B. Asbury, R. J. Ellingson, H. N. Ghosh, S. Ferrere, A. J. Nozik, and T. Q. Lian, J. Phys. Chem. B 103, 3110-3119 (1999).

13. J. B. Asbury, E. Hao, Y. Wang, and T. Q. Lian, J. Phys. Chem. B, 104, 11957-11964 (2000)

14. G. Benkö, J. Kallioinen, J. Korppi-Tommola, A. P. Yartsev, and V. Sundström, J. Am. Chem. Soc. 124, 489-493 (2002).

15. J. Kallioinen, G. Benkö, P. Myllyperkiö, L. Khriachtchev, B. Skarman, R. Wallenberg, M. Tuomikoski, J. KorppiTommola, V. Sundström, and A. P. Yartsev, J. Phys. Chem. B 108, 6365-6373 (2004).

16. S. Pelet, M. Grätzel, and J. E. Moser, J. Phys. Chem. B 107, 3215-3224 (2003).

17. J. Moser, M. Grätzel, D. K. Sharma, and N. Serpone, Helv. Chim. Acta, 68, 1686-1690 (1985).

18. S. A. Haque, E. Palomares, B. M. Cho, A. N. M. Green, N. Hirata, D. R. Klug, and J. R. Durrant, J. Am. Chem. Soc. 127, 3456-3462 (2005).

19. B. Wenger, M. Grätzel, and J. E. Moser, J. Am. Chem. Soc. 127, 12150-12151 (2005).

20. J. E. Moser, P. Bonhôte, and M. Grätzel, Coord. Chem. Rev. 171, 245-250 (1998).

21. J. E. Moser, and M. Grätzel, Chimia 52, 160-162 (1998).

22. J. E. Moser, M. Wolf, F. Lenzmann, and M. Grätzel, Zeit. Phys. Chem. 212, 85-92 (1999).

23. C. Zimmermann, F. Willig, S. Ramakrishna, B. Burfeindt, B. Pettinger, R. Eichberger, and W. Storck, J. Phys. Chem. B 105, 9245-9253 (2001).

24. R. Huber, J. E. Moser, M. Grätzel, and J. Wachtveitl, Proc. SPIE, 5223, 121-131 (2003).

25. G. Benkö, J. Kallioinen, P. Myllyperkiö, F. Trif, J. E. I. Korppi-Tommola, A. P. Yartsev, and V. Sundström, J. Phys. Chem. B 108, 2862-2867 (2004).

26. N. A. Anderson, X. Ai, D. T. Chen, D. L. Mohler, and T. Q. Lian, J. Phys. Chem. B 107, 14231-14239 (2003).

27. E. Galoppini, Coord. Chem. Rev. 248, 1283-1297 (2004).

28. P. Piotrowiak, E. Galoppini, Q. Wei, G. J. Meyer, and R. Wiewior, J. Am. Chem. Soc. 125, 5278-5279 (2003).

29. L. Gundlach, S. Felber, W. Storck, E. Galoppini, Q. Wei, and F. Willig, Res. Chem. Intermed. 31, 39-46 (2005).

30. R. Ernstoffer, S. Felber, W. Storck, E. Galoppini, Q. Wei, and F. Willig, Res. Chem. Intermed. 31, 643-647 (2005).

31. G. R. R. A. Kumara, K. Tennakone, V. P. S. Perera, A. Konno, S. Kaneko, and M. Okuya, J. Phys. D: Appl. Phys. 34, 868-873 (2001).

32. A. Kay and M. Grätzel, Chem. Mater. 14, 2930-2935 (2002).

33. E. Palomares, J. Clifford, S. A. Haque, T. Lutz, and J. R. Durrant, J. Am. Chem. Soc. 125, 475-482 (2003).

34. M. K. Nazeeruddin, P. Pechy, T. Renouard, S. M. Zakeeruddin, R. Humphry-Baker, P. Comte, P. Liska, E. Costa, V. Shklover, L. Spiccia, G. B. Deacon, C. A. Bignozzi, and M. Grätzel, J. Am. Chem. Soc. 123, 1613-1624 (2001).

35. H. Nusbaumer, J. E. Moser, S. M. Zakeeruddin, M. K. Nazeeruddin, and M. Grätzel, J. Phys. Chem. B 105, 1046110464 (2001).

36. P. F. Barbara, T. J. Meyer, and M. A. Ratner, J. Phys. Chem. 100, 13148-13168 (1996).

37. D. M. Adams, L. Brus, C. E. D. Chidsey, S. Creager, C. Creutz, C. R. Kagan, P. V. Kamat, M. Lieberman, S. Lindsay, R. A. Marcus, R. M. Metzger, M. E. Michel-Beyerle, J. R. Miller, M. D. Newton, D. R. Rolison, O. Sankey, K. S. Schanze, J. Yardley, and X. Zhu, J. Phys. Chem. B 107, 6668-6697 (2003). 
38. M. Kondo, T. Tada, and K. Yoshizawa, J. Phys. Chem. A 108, 9143-9149 (2004).

39. D. Vanmaekelbergh, Electron Transfer at electrodes and Interfaces, In Electron Transfer in Chemistry, V. Balzani, Ed., Wiley-VCH, Weinheim, 2001; Vol. 1, pp 126-188.

40. W. Rippard, A. Perella, F. Albert, and R. Buhrman, Phys. Rev. Lett. 88, 046805 (2002). 